Revue des patrimoines

\title{
La fresque sur ciment : un renouveau artistique
}

Marine Bayle, Éléonore Gueit, Évelyne Darque-Ceretti, Matthieu Horgnies et Marc Aucouturier

\section{OpenEdition}

\section{Journals}

Édition électronique

URL : http://journals.openedition.org/insitu/10813

DOI : 10.4000/insitu. 10813

ISSN : 1630-7305

\section{Éditeur}

Ministère de la culture

\section{Référence électronique}

Marine Bayle, Éléonore Gueit, Évelyne Darque-Ceretti, Matthieu Horgnies et Marc Aucouturier, « La fresque sur ciment : un renouveau artistique », In Situ [En ligne], 22 | 2013, mis en ligne le 14 novembre 2013, consulté le 03 mai 2019. URL : http://journals.openedition.org/insitu/10813 ; DOI : 10.4000/ insitu. 10813

\section{Ce document a été généré automatiquement le 3 mai 2019.}

\section{(c) $(1) \odot$}

In Situ Revues des patrimoines est mis à disposition selon les termes de la licence Creative Commons Attribution - Pas d'Utilisation Commerciale - Pas de Modification 4.0 International. 


\title{
La fresque sur ciment : un renouveau artistique
}

\author{
Marine Bayle, Éléonore Gueit, Évelyne Darque-Ceretti, Matthieu Horgnies \\ et Marc Aucouturier
}

\section{Remerciements}

Merci à Mme Solène Delahousse, artiste fresquiste, pour son aide précieuse et ses conseils tout autant artistiques que techniques. Merci aussi à Mme Catherine Bouillon (Lafarge) pour son aide durant certaines analyses par microscopie électronique à balayage. Les auteurs remercient également M. R. de la Soujeole et Mme C. Garguelle pour l'accès aux œuvres de la fresque de saint Hippolyte et du Chemin de croix de Fourqueux.

1 Depuis les premières œuvres pariétales, la peinture murale est un art qui a évolué et qui s'exécute aujourd'hui selon de nombreuses techniques acquises au cours des siècles. L'une d'entre elles, la technique de peinture à fresque, est certainement la plus pérenne. Sa réalisation sur enduit frais d'où provient le terme 'a fresco' procure longévité mais soumet l'artiste à une palette de couleurs restreinte ainsi qu'à de nombreuses difficultés de réalisation. Traditionnellement sur enduit de chaux, la fresque connaît un renouveau grâce à l'utilisation de ciment moderne au début du $\mathrm{XX}^{\mathrm{e}}$ siècle. Henri Marret, artiste pluridisciplinaire dans l'art du dessin et de la peinture, s'est passionné pour la technique à fresque et a participé à son évolution en l'adaptant aux matériaux de l'ère industrielle.

\section{La technique de peinture murale à fresque}

2 La technique de peinture à fresque a la particularité de traverser les époques parfois sans connaître d'altération. Les pigments, en suspension dans l'eau, sont appliqués au pinceau directement sur un enduit frais. D'origine minérale, les grains de pigments se fixent et adhèrent au support lors de la prise de l'enduit. La préparation des enduits supports et l'application précise des pigments sont aux fondements de la longévité de l'œuvre. Chaque fresquiste dépose son empreinte artistique et procure ainsi un caractère particulier, unique et irréversible à la peinture murale. 
3 La préparation du support de travail est un élément indispensable qui conditionne la réalisation. Le mur d'accroche doit être sain et propre car une succession de couches d'enduits de granulométrie décroissante est appliquée afin d'obtenir un réservoir suffisant d'humidité. Si le nombre de couches peut atteindre sept, la fresque peut se contenter de deux ou trois enduits majeurs. Le premier appliqué sur le mur est une couche de préparation ou gobetis qui favorise le maintien des enduits suivants. Puis l'artiste applique l'arricio et enfin l'intonaco qui sera peint à frais. Parfois le fresquiste décide d'ajouter une couche encore plus fine, l'intonachino. L'épaisseur de chacun des enduits est régie par la granulométrie de la charge employée; elle sera d'une fois et demie la taille maximale du grain ${ }^{1}$ (fig. $\mathbf{n}^{\circ} \mathbf{1}$ ).

Figure 1

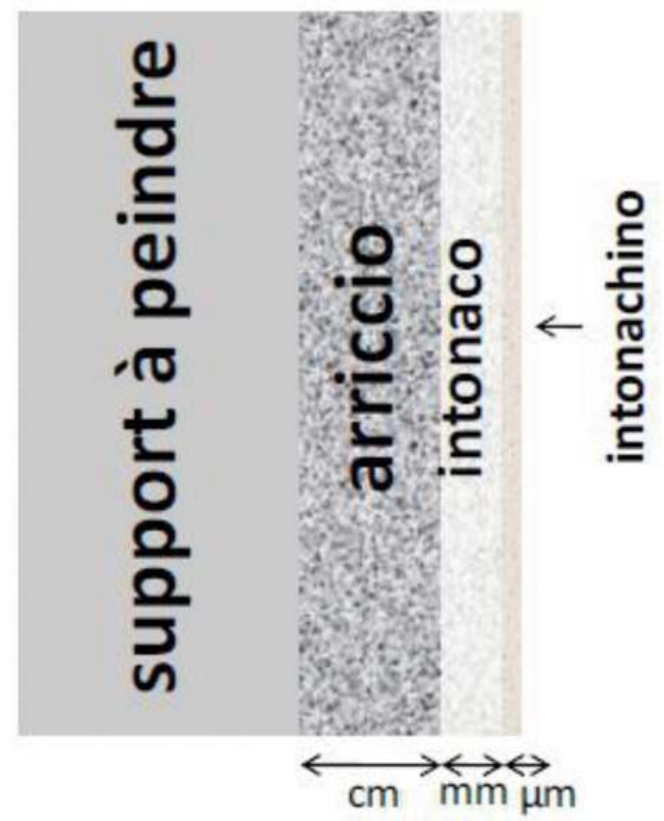

Superposition classique des couches d'enduits lors de la réalisation d'une fresque et ordre de grandeur des épaisseurs.

Thèse de Mines-Paris Tech, Gueit, Éléonore, 2012.

4 La technique de peinture à fresque ne peut s'exécuter sans une phase de préparation artistique aboutie. En effet, une fois appliqués, les pigments s'insèrent de façon irréversible dans l'enduit. Seule une détérioration de la partie manquée permettra de corriger l'œuvre. Il est donc primordial que l'artiste avance dans sa réalisation en connaissant à l'avance chacune des couleurs qu'il souhaite appliquer et dans quel ordre. Un dessin préparatoire de la taille de la fresque est mis au point. Ce carton permet de dessiner sur l'arricio les grandes lignes de la fresque. Ce tracé appelé sinopia de par l'utilisation traditionnelle d'ocre rouge, délimite les journées de travail ou giornata. Petit à petit l'intonaco recouvre l'arricio et le carton, percé de trous sur les contours de l'esquisse, est positionné sur l'enduit frais prêt à être coloré. À l'aide de la poncette (petit sac de 
pigments secs) l'artiste tamponne les détails qu'il souhaite retrouver sur le mur. Une fois reliés au pinceau, ces pointillés donnent au fresquiste les délimitations de couleur².

\section{La fresque sur chaux}

5 La chaux est le matériau de base dans la technique de fresque classique. Elle est obtenue après cuisson et extinction de la pierre calcaire extraite dans des carrières. L'oxyde de calcium formé, aussi appelé chaux vive, est un composé instable. Au contact de l'eau, il permet d'obtenir la chaux éteinte ou hydroxyde de calcium. Sous l'action du dioxyde de carbone présent dans l'atmosphère, l'hydroxyde de calcium réagit pour donner du carbonate de calcium et de l'eau. La chaux aérienne ne subit cette réaction qu'au contact de l'air alors que la chaux hydraulique réagit d'abord à l'eau puis ensuite à l'air. Ce phénomène chimique a toujours été considéré comme fondement de la fresque et permet d'apporter dureté et solidité à l'œuvre 3 .

6 Si les plus anciennes œuvres à fresque sur chaux sont datées de l'époque Minoenne, entre 1900 et $1400 \mathrm{av}$. JC, la période Romaine (VIIe av. JC - IVe ap. JC) conduit la technique à son apogée. Durant les siècles romains, quatre styles se succèdent dans lesquels la fresque connaît des évolutions primordiales. Des textes comme ceux de Vitruve, De Architectura ${ }^{4}$, ou de Pline l'Ancien, Naturalis Historiae ${ }^{5}$, sont aujourd'hui de précieux témoignages des caractéristiques techniques de l'art. La fresque romaine présente la particularité du lissage des enduits essentiellement dans le premier style. À l'apogée de la technique, l'artiste devait très certainement appliquer l'enduit sur son support puis faire remonter l'humidité chargée en hydroxyde de calcium à la surface en lissant l'enduit. Puis, les pigments sont appliqués parfois même sous la forme d'un badigeon, c'est-à-dire liés à une quantité importante de chaux lorsque l'enduit présente une porosité d'accroche trop faible. Un polissage final de la surface favorisant l'insertion des pigments ou du badigeon permet d'obtenir un aspect parfaitement lisse et plus ou moins brillant.

7 La fresque a ensuite évolué au cours des siècles donnant naissance sous l'époque romane à des techniques mixtes utilisant des liants organiques et une palette de couleurs plus fournie. Si le rendu immédiat des fresques a pu être exceptionnel, la réaction de la chaux (basique par nature) avec les éléments organiques utilisés a réduit le nombre d'œuvres encore aujourd'hui en bon état de conservation. Sous l'ère gothique, la technique de fresque a été peu à peu délaissée. Mais, c'est à la Renaissance que les fondements sont retrouvés avec le Traité des Arts ${ }^{6}$ de Cennini, aujourd'hui référence majeure. Jusqu'à nos jours de nombreux artistes n'ont cessé de faire perdurer cette technique selon leur créativité et les possibilités offertes par les matériaux et les pigments 7 .

\section{L'apparition de la fresque sur ciment}

8 Puisque le fondement de la technique réside dans l'application de pigments sur un enduit frais, il est théoriquement possible de peindre à fresque sur tout autre support frais que la chaux aérienne, pourvu que celui-ci durcisse dans des délais raisonnables. Lorsque l'emploi des bétons commence à se généraliser au $\mathrm{XX}^{\mathrm{e}}$ siècle, certains artistes font le pari de peindre à fresque sur des enduits de chaux hydraulique, voire de ciment ${ }^{8}$.

En France, dans les années 1920, Henri-Marcel Magne, professeur au Conservatoire National des Arts et Métiers, développe la technique suite aux observations d'incuits détériorant les œuvres sur chaux. Selon lui, la solidité de la fresque ne repose pas tant sur 
la carbonatation que sur la pénétration des pigments dans la capillarité de l'enduit. Il réalise avec succès plusieurs séries d'essais sur ciment dans son atelier et se voit finalement confier la réalisation de la frise extérieure de l'église Saint-Christophe-deJavel à Paris, peinte en respectant les caractéristiques traditionnelles des pigments et des outils de la fresque mais sur enduit de ciment. Parallèlement, le peintre Nîmois Henri Pertuis mène ses propres essais de fresque sur chaux hydraulique et constate qu'en dépit des préjugés défavorables à ce sujet, ses résultats sont tout-à-fait concluants. Henri Marret fait partie de ces artistes novateurs qui expérimentent la fresque sur enduits de chaux hydraulique ou de ciment qu'il préfère aux enduits de chaux aérienne pour les œuvres extérieures en raison de leur plus grande dureté. Peu d'artistes se sont adonnés à ce type de fresque, Henri-Marcel Magne, Henri Marret, Henri Pertuis et également Jean Souverbie ont choisi de développer l'art de la peinture murale à frais sur ciment ${ }^{9}$, malgré les oppositions de leurs confrères persuadés de l'impossibilité de la technique. On retrouve également des muralistes mexicains tels Diego Rivera et David Alfaro Siqueiros ${ }^{10}$.

Certains fresquistes ont laissé des sources écrites sur leur pratique de la peinture à frais sur ciment et s'accordent sur le fait que techniquement, elle diffère peu de la fresque traditionnelle. La palette est la même que pour la fresque sur chaux: des pigments minéraux capables de résister au contact du mortier frais ${ }^{11}$. Par ailleurs, l'artiste doit composer avec la teinte de son enduit : sur un ciment gris, les couleurs n'ont pas le même aspect que sur une chaux ou un ciment blanc ${ }^{12}$. Concernant les liants, chaque artiste a sa propre vision des choses. Par analogie avec la fresque à la chaux, Magne utilise une "eau de ciment". Siqueiros, lui, se tourne parfois vers des produits organiques. Une étude scientifique de la fresque America Tropical située à Los Angeles révèle que les pigments ont probablement été dilués dans de la nitrocellulose avant d'être appliqués sur l'enduit de ciment frais. Par un double effet chimique et physique, la mise en contact du liant organique et de l'enduit aqueux a immédiatement posé des problèmes de compatibilité et de dégradation du liant, et la couche picturale a aujourd'hui quasiment disparu ${ }^{13}$.

11 La réalisation de l'œuvre en elle-même suit le modèle de la fresque traditionnelle : dessin préparatoire ${ }^{14}$ puis application de l'enduit frais et des pigments sur des zones restreintes. Afin de gagner du temps de travail sur son œuvre, Siqueiros, gêné par la prise rapide de ses enduits, développe un système de pistolet à air qui lui permet de propulser très rapidement ses pigments sur la surface avant qu'elle ne durcisse ${ }^{15}$. Malgré les remarques sceptiques de certains artistes et scientifiques concernant la technique de fresque sur ciment selon la technique ancestrale, Henri Marret, passionné par cet art, nous a laissé un patrimoine de réalisations magnifiques et durables.

\section{Descriptions des œuvres de Marret : entre chaux et ciment}

Artiste pluridisciplinaire, Henri Marret (1878-1964) réalise de nombreuses œuvres comme des aquarelles, des peintures et des gravures. Mais, il s'illustre tout particulièrement à l'art de peinture à fresque. Initié par Paul Baudouin en 1912, il maîtrise cette technique à la perfection et la développe. Il travaille un nouveau type d'enduit pour l'époque, le ciment Portland. La communication qu'il rédige dans le bulletin technique de Lafarge ${ }^{16}$ ("Société anonyme des Chaux et Ciments de Lafarge et du Teil ») nous montre son intérêt particulier au ciment. Il réalise alors simultanément des œuvres sur enduit de chaux et enduit de ciment. 

techniques mais aussi d'apporter des informations essentielles sur l'art de la fresque sur ciment au XXe siècle. La présente étude met en opposition le Chemin de Croix de l'église Sainte Croix de Fourqueux sur chaux avec la fresque des Martyrs de saint Hippolyte et de saint Laurent sur enduit de ciment gris.

\section{Chemin de croix de l'église Sainte croix de Fourqueux}

\section{Figure 2}

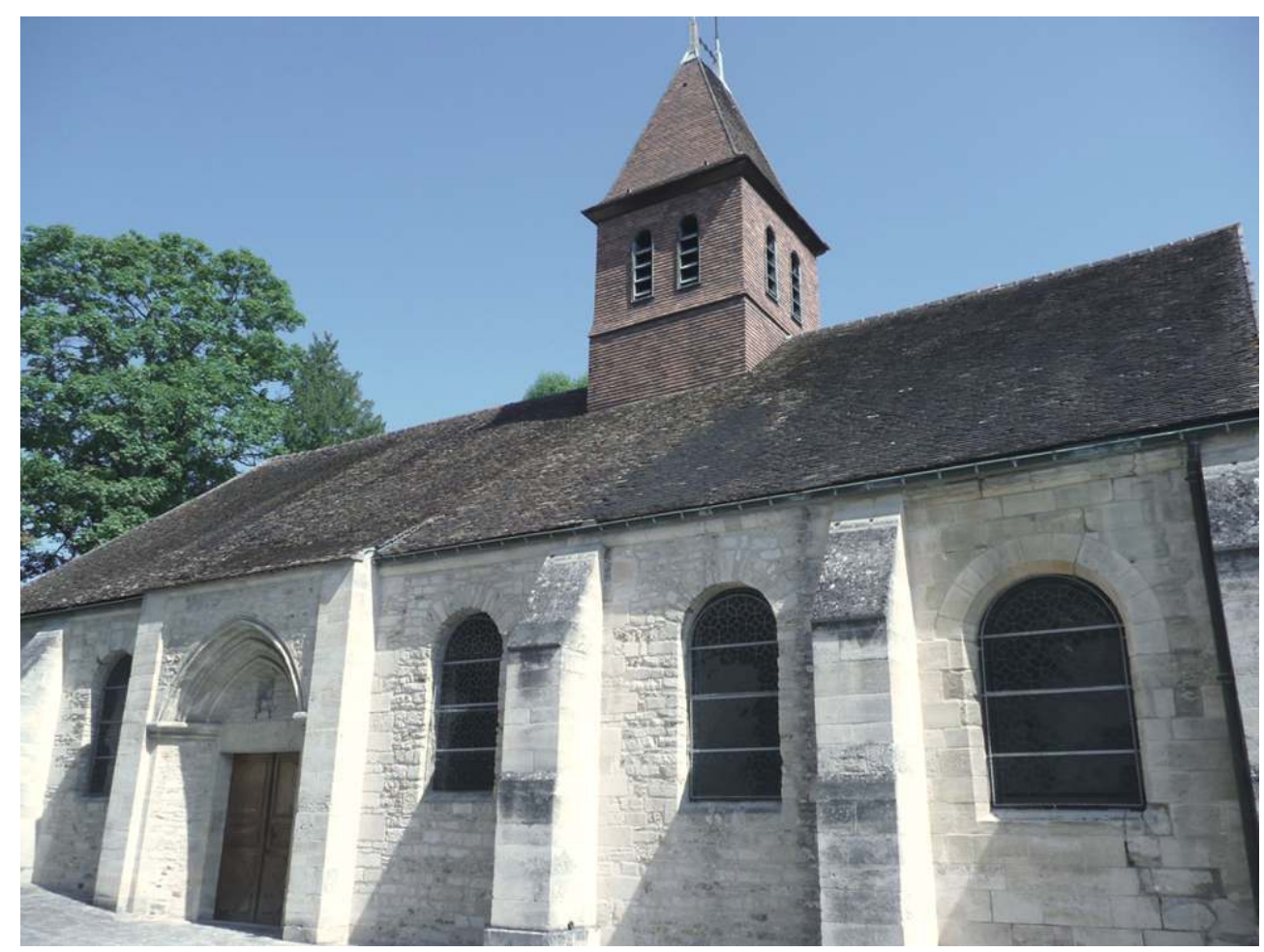

Vue extérieure de l'église Sainte Croix de Fourqueux.

PHOT. BAYLE, MARINE, 2011. ( ) M. BAYLE.

La maison familiale de Marret se trouve à Fourqueux, ville des Yvelines (78), dont il est maire de 1937 à 1944. L'artiste réalise de nombreuses œuvres pour sa ville au sein de l'église Sainte Croix (fig. $\mathbf{n}^{\circ}$ 2). À l'intérieur, on retrouve une imposante fresque sur chaux représentant un Monument aux Morts, peinte sur le mur du fond de l'église. En circulant le long des murs internes de l'édifice, il est indispensable d'admirer les 14 panneaux du Chemin de Croix peints à fresque en 1922. Henri Marret a réalisé de nombreux chemins de croix et le modèle employé dans l'église de Fourqueux a été repris à plusieurs reprises pour les églises de Fresnoy-lès-Roye ou Irles dans la Somme (80). Le chemin de Croix portatif de Fourqueux consiste en 12 panneaux carrés de $54,4 \times 54,4 \mathrm{~cm}^{2}$ et deux rectangulaires de $55,5 \times 187 \mathrm{~cm}^{2}$ peints à fresque sur chaux aérienne. Tous sont exécutés sur un support en béton armé et placés dans des cadres en bois (fig. $\mathbf{n}^{\circ} \mathbf{3}$ ). La multiple réalisation de cette fresque montre que Marret avait préparé un carton de son œuvre qu'il pouvait ainsi répéter autant de fois qu'il le souhaitait selon les commandes qu'il recevait. L'utilisation d'un carton percé de trous ou poncif se remarque parfois sur 
l'œuvre, comme ici sur l'un des panneaux du Chemin de Croix, lorsque des pointillés n'ont pas été dissimulés par la couleur lors de la réalisation (fig. $\left.\mathbf{n}^{\circ} \mathbf{4}\right)$.

Figure 3

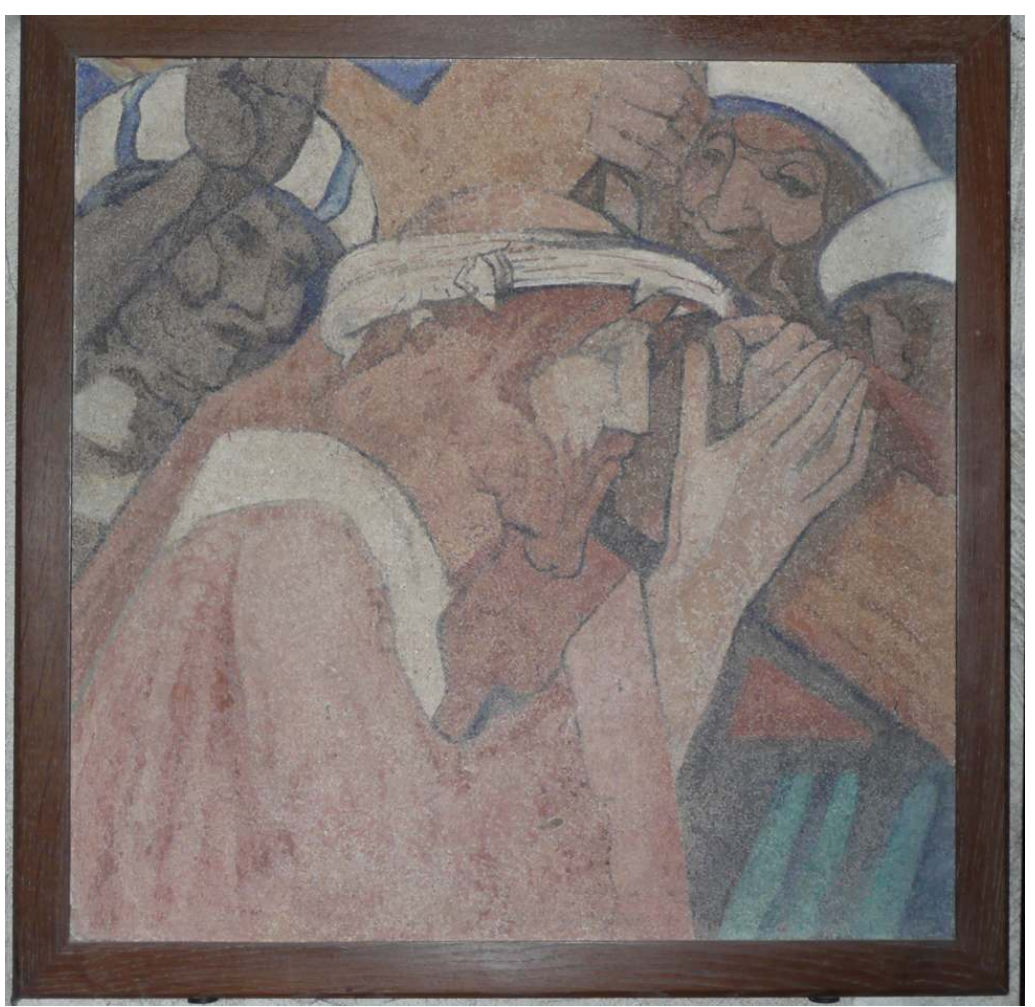

Station II du Chemin de croix de Fourqueux, par Henri Marret.

Phot. Bayle, Marine, 2011. (c) M. Bayle.

Figure 4

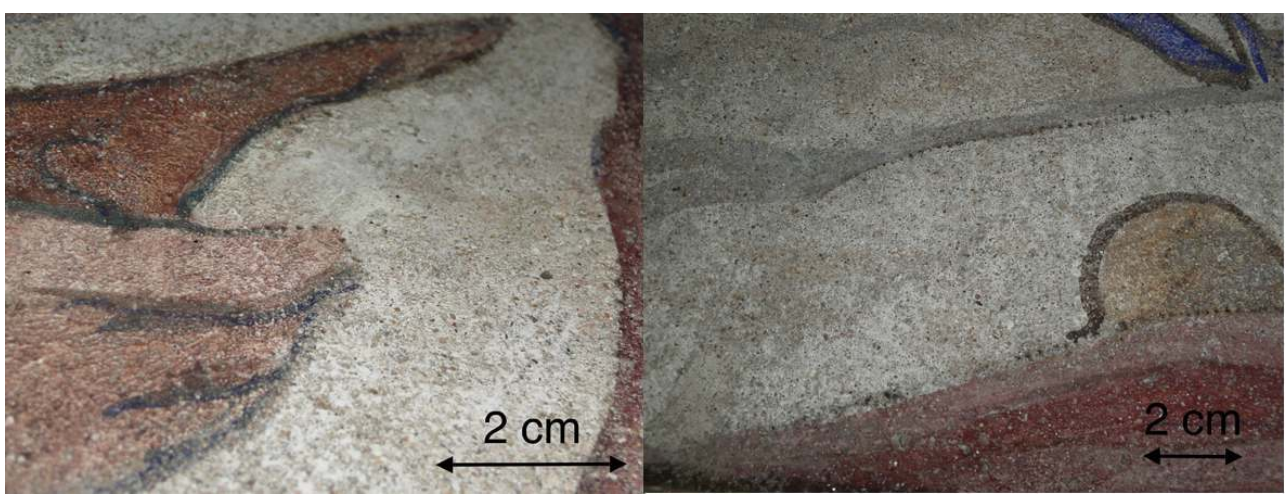

Tracé du poncif sur le Chemin de Croix de Fourqueux. 4a : détail d'un doigt de femme, station VIII, Jésus rencontre les femmes de Jérusalem. $4 \mathrm{~b}$ : détail au niveau de la tunique et du pied de Jésus, station IX, Jésus tombe pour la troisième fois.

PHOT. BAYLE, MARINE, 2011. ( $)$ M. BAYLE. 


\section{Martyrs de saint Hippolyte et de saint Laurent}

$15 \mathrm{Au} \mathrm{XIX}^{\mathrm{e}}$ siècle, les scientifiques et les industriels tentent de retrouver la structure du ciment romain composé d'un mélange de gypse (sulfate de calcium hydraté), de silice, de pouzzolane et de carbonate de calcium et de l'améliorer afin d'obtenir un liant hydraulique solide et industrialisable ${ }^{17}$. Le ciment Portland prend naissance et provient d'un mélange de chaux, de silice, d'alumine et d'oxydes de fer. Ce mélange calcaire-argiles est cuit à haute température $\left(1400^{\circ} \mathrm{C}\right)$ afin d'obtenir le clinker, petites billes solides dont le broyage fournit une poudre appelée ciment. Mélangé à de l'eau, le ciment réagit pour former une pâte solide en quelques heures qui devient particulièrement résistante après quelques jours ${ }^{18}$.

\section{Figure 5}

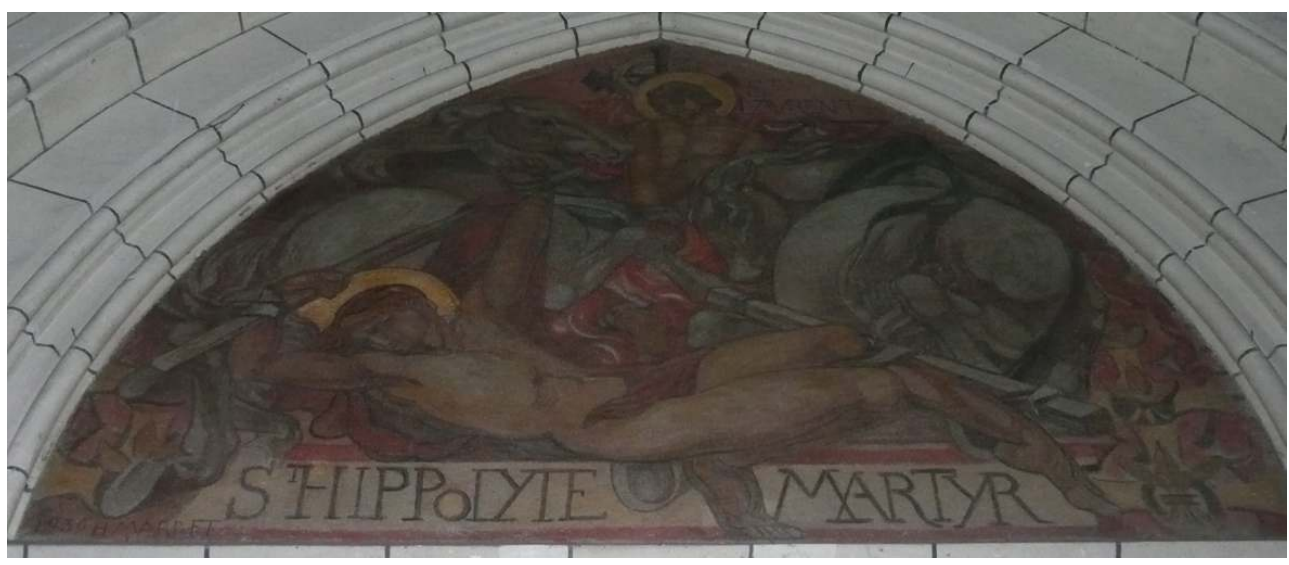

Fresque des Martyrs de saint Hippolyte et de saint Laurent d'Henri Marret.

Phot. Bayle, Marine, 2011. (c) M. Bayle.

Henri Marret choisit d'utiliser ce matériau moderne et réalise la fresque extérieure des Martyrs de saint Hippolyte et de saint Laurent sur enduit de ciment gris en 1936 sur le fronton de l'église Saint Hippolyte dans le XIII ${ }^{e}$ arrondissement de Paris. Protégée sous un porche, elle représente une scène de torture violente où saint Hippolyte placé au centre, est tiré par des chevaux alors que saint Laurent se trouve plus en arrière à droite, sur une grille ardente. Dans les tons rouges rappelant la chaleur du feu et la souffrance des martyrs, Marret a su tirer profit de la couleur grise de l'enduit notamment pour le dessin des chevaux à fresque (fig. $\mathbf{n}^{\circ}$ ) $)$. Tout comme pour le Chemin de Croix, des indices montrent que la réalisation s'est effectuée sur enduit frais. La fresque est en demi-cercle de base $216 \mathrm{~cm}$ pour une hauteur maximale de $160 \mathrm{~cm}$. Malgré la réputation de Marret comme étant un artiste très rapide, il est impossible de peindre à fresque une surface si grande en une seule fois avec de plus des conditions d'accès difficiles (fig. $\mathbf{n}^{\circ} \mathbf{6}$ ). Au moins une démarcation de travail de l'enduit est visible. Habituellement placées selon les contours, les giornate de Marret ont la particularité de traverser la fresque sans suivre de décors, mis à part le dos d'un des chevaux associés à saint Hippolyte. Par ailleurs, la présence de traces de doigts appartenant à Marret ou très certainement au maçon qui lui préparait les enduits, montre bien la difficulté de travailler à frais sur une peinture murale (fig. $\mathbf{n}^{\circ} 7$ ). 
Figure 6

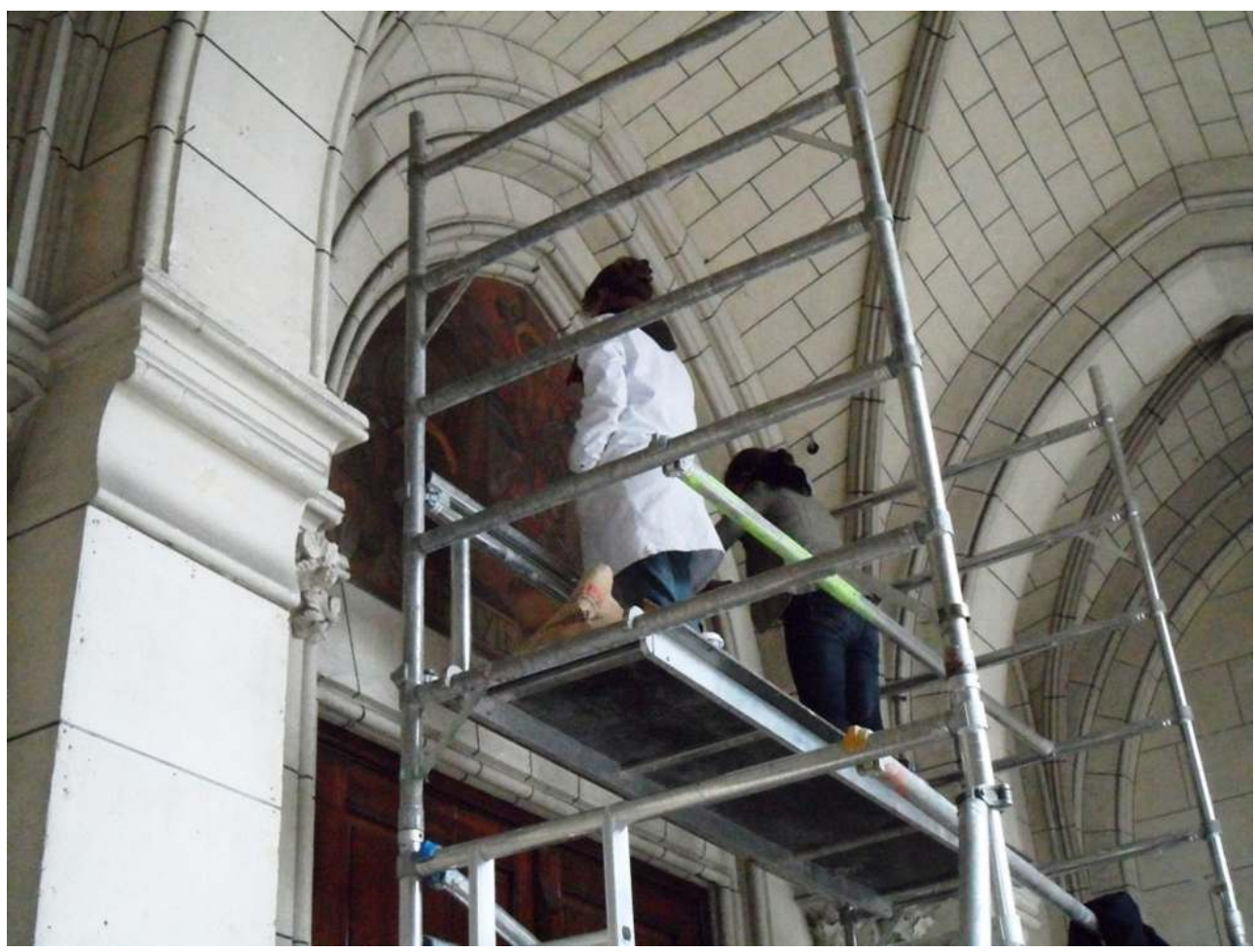

Observation et prélèvement d'échantillons sur la fresque des Martyrs de saint Hippolyte et de saint Laurent d'Henri Marret.

Phot. Aucouturier, Marc, 2011. (c) M. Aucouturier.

Figure 7

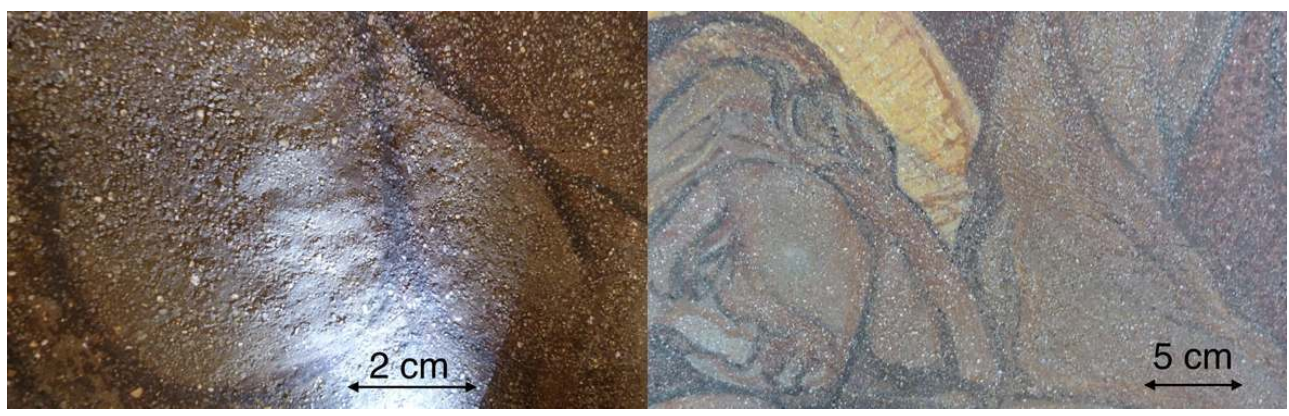

Détails de l'enduit de ciment sur la fresque des Martyrs de saint Hippolyte et de saint Laurent d'Henri Marret. $7 a$ : Traces de doigts dans l'enduit. $7 \mathrm{~b}$ : Délimitation dans l'enduit (giornata), détail au niveau du corps de saint Hippolyte.

Phot. Gueit, Éléonore, 2011. (c) É. Gueit.

\section{Étude des fresques de Marret : comparaisons}

17 Ces deux peintures à fresque de Marret ont été étudiées au Centre de Recherche et de Restauration des Musées de France (C2RMF) et au Centre de Recherche de Lafarge (LCR) afin de comprendre les particularités de la technique sur enduit de ciment. 


\section{Description des enduits durcis}

Henri Marret nous a offert des exemples de fresques sur enduits de chaux aérienne et de ciment gris. Malgré des caractéristiques proches dues à sa technique de réalisation, la prise de ces enduits est différente et apporte des difficultés de travail. Henri Marret utilise dans les deux cas, sur chaux ou sur ciment, un enduit très grossier chargé de grains de sable de carrière non uniformes et de tailles irrégulières. L'affleurement des grains en surface, tout particulièrement sur la fresque de Saint Hippolyte (sur plusieurs centaines de $\mu \mathrm{m}$ ), procure une profondeur aux œuvres. Le sable utilisé pour le Chemin de Croix donne un aspect moins agressif car la surface reste plane et relativement lisse (rugosité moyenne de l'ordre d'une centaine de $\mu \mathrm{m}$ ) (fig. $\left.\mathbf{n}^{\mathbf{0}} \mathbf{8}\right)$; cette observation montre que Marret a travaillé l'enduit de sa fresque d'une façon toute particulière par lissage. Marret a très certainement utilisé une taloche bois, outil classique pour l'époque de réalisation, afin de préparer son enduit de surface tel un arricio, normalement avant dernière couche donc chargé plus grossièrement. Puis, c'est en laissant la chaux au repos que l'humidité remonte en surface sous la forme d'une couche d'eau laiteuse. À ce moment-là, Marret utilise une éponge humidifiée et très essorée pour récupérer la couche d'eau de chaux formée. Les grains de sable utilisés en tant que charge ne sont donc pas recouverts de pâte et sont directement visibles à la surface. La couleur peut ensuite être appliquée au pinceau.

Figure 8
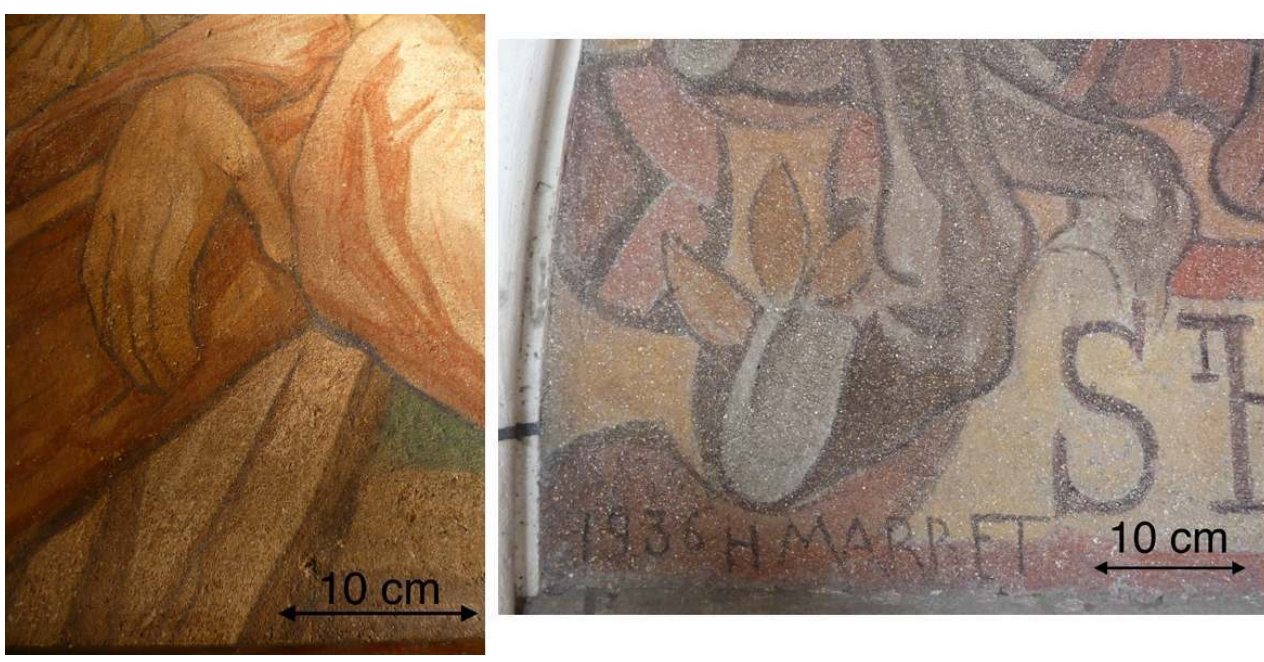

Détails de la surface des fresques de Marret. 8a : Détail de la station VIII du Chemin de croix de Fourqueux, mains de Jésus. 8b : Signature de Marret sur la fresque de saint Hippolyte montrant l'affleurement important des grains de sable en surface.

PHOT. BAYLE, MARINE, 2011. (C) M. BAYLE.

Pour la fresque sur ciment de saint Hippolyte, le travail de l'enduit semble tout à fait différent. Malgré un enduit chargé en gros grains, la technique de lissage observée sur le Chemin de Croix sur chaux ne semble pas avoir été employée. Le grain est visible en surface et le toucher est très rugueux. L'affleurement des grains montre un dépôt simple de l'enduit à la taloche. Certaines zones, plus travaillées, comme l'auréole de saint Hippolyte ainsi que les visages, ont un rendu plus lisse avec une densité de grains moins importante (fig. $\mathbf{n}^{\circ} \mathbf{9}$ ). L'observation de prélèvements d'enduits en stratigraphie sous 
microscopie optique et microscopie électronique à balayage au C2RMF montre que non seulement la surface possède de gros grains de sable, d'une taille parfois très supérieure au millimètre, mais que l'enduit contient des cendres ainsi que des charges colorées, jaunes. Ces charges présentes dans la masse sont détectées par des mesures colorimétriques et procurent une teinte orangée au ciment (fig. $\left.\mathbf{n}^{\circ} \mathbf{1 0}\right)$.

Figure 9

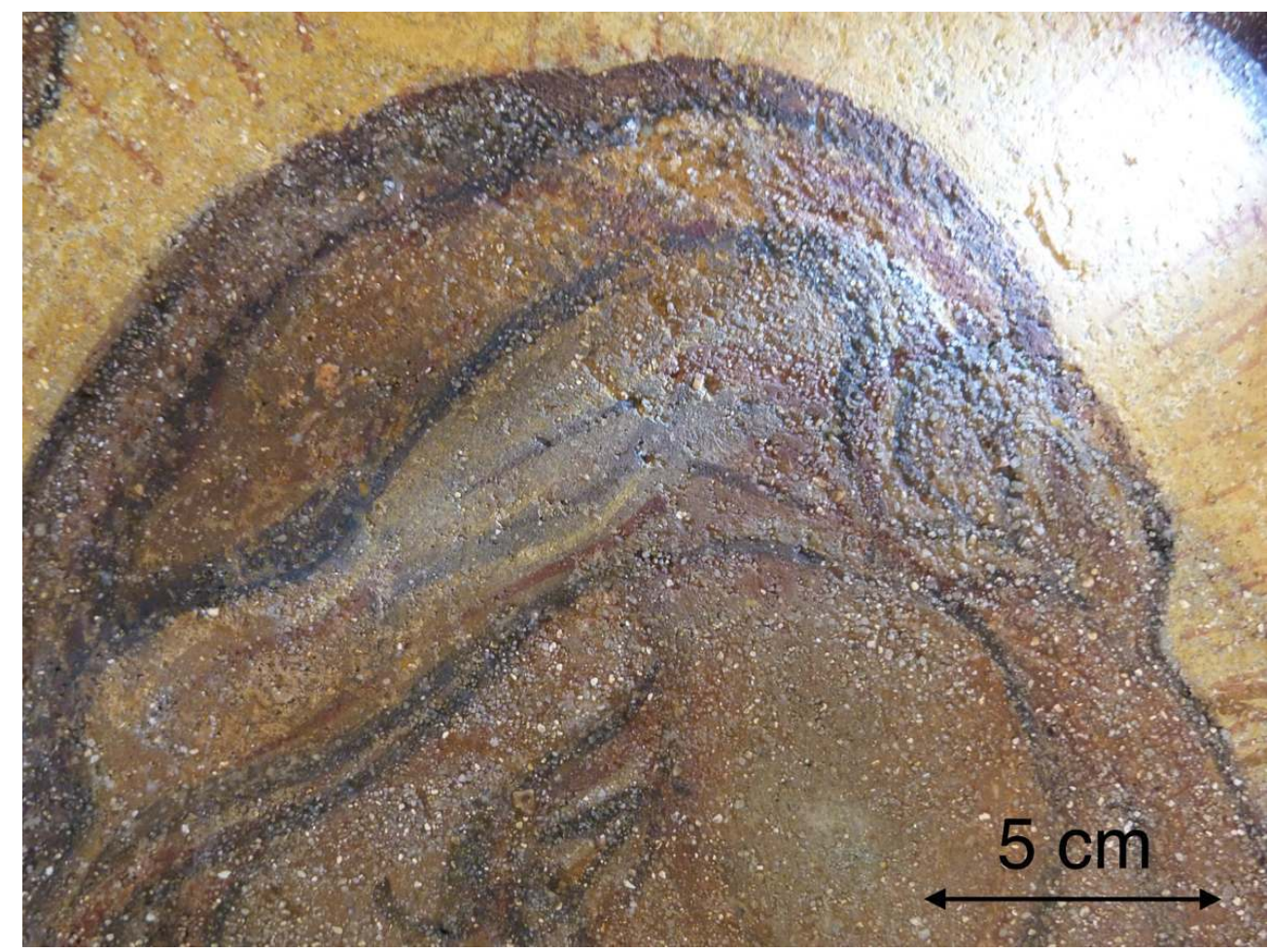

Détails de l'auréole et des cheveux de saint Hippolyte où l'enduit de ciment est moins grossier et plus travaillé.

Phot. Gueit, Éléonore, 2011. (c) É. Gueit. 


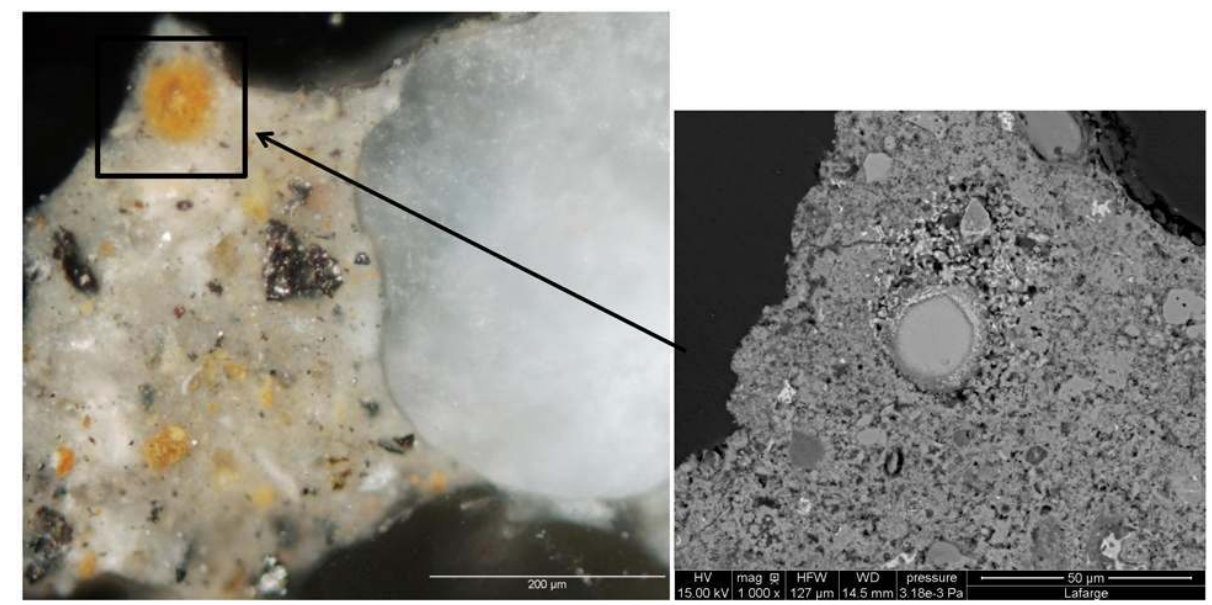

Coupe stratigraphique du ciment de la fresque de saint Hippolyte observée sous microscopies optique et électronique à balayage (mode électrons rétrodiffusés). 10a : Coupe observée en microscopie optique. $10 \mathrm{~b}$ : Coupe observée en microscopie électronique à balayage, mode électrons rétrodiffusés.

(c) Bayle, Marine/C2RMF, LCR, 2011.

Le ciment est un matériau dont la prise, relativement rapide, se fait sous l'action de l'eau contrairement à la chaux. Il est alors évident que le travail de cet enduit ne peut pas être effectué comme pour le Chemin de Croix de Fourqueux. L'action de lissage ne peut pas avoir un effet similaire et peut même être destructrice. D'ailleurs, Georges Lecaron, dans son Essai sur des techniques de peinture: La fresque ${ }^{19}$, doute de la technique de fresque sur ciment en expliquant que le lissage ne peut pas être effectué en raison d'une prise trop rapide. La définition de fresque ne parle pas de lissage de l'enduit. Bien évidemment, celui-ci doit pouvoir être travaillé et aplani pour présenter une surface esthétique mais le lissage typiquement romain ne semble apporter que plus de solidité et une apparence plus brillante à l'œuvre.

21 Tout comme la chaux, le travail à frais sur ciment doit donc s'exécuter sous des contraintes de temps et de travail préparatoire précis, étant donné que le ciment Portland humidifié s'hydrate et durcit après seulement une à deux heures (en fonction des conditions ambiantes). C'est en cela que réside la différence essentielle avec la prise de la chaux. Alors que la chaux se carbonate au contact du dioxyde de carbone présent dans l'atmosphère, le ciment Portland réagit ("s'hydrate») au contact de l'eau pour transformer les silicates tricalciques (alite) et bicalciques (belite) en silicates de calcium hydratés $(\mathrm{C}-\mathrm{S}-\mathrm{H})$ et en portlandite $(\mathrm{Ca}(\mathrm{OH}) 2)^{20}$. De plus, certains composés mineurs du ciment tels que les aluminates de calcium peuvent réagir trop rapidement (prise " flash » qui amoindrit dramatiquement la résistance mécanique). Une petite quantité de gypse est donc toujours ajoutée au clinker lors de la préparation du ciment pour former un peu de monosulfate de calcium hydraté et limiter la prise lors des premières minutes de malaxage avec l'eau.

Malgré tout, le travail de la surface de la pâte de ciment fraîche reste difficile à modéliser car il dépend de nombreux paramètres tels que l'humidité relative de l'atmosphère, la température et surtout des proportions du mélange réalisé (rapport eau/ciment). Mais, 
contrairement à la chaux qui réagit au contact du dioxyde de carbone de la surface (en contact avec l'atmosphère) jusqu'au cœur, le ciment réalise une prise en masse. Par expérience, Henri Marret a ainsi pu tirer profit des différences apportées par un enduit de ciment - coloration grise, aspect brut et granuleux, prise rapide - pour appliquer à frais des pigments au pinceau.

\section{Couleur des fresques et usage des pigments}

Grâce aux analyses réalisées au C2RMF, la palette de Marret a été déterminée. Formé à l'art de la fresque et respectueux de la technique, il a utilisé des pigments minéraux classiques de la fresque que l'on retrouve abondamment au début $\mathrm{du} \mathrm{XX}^{\mathrm{e}}$ siècle. La fresque sur enduit de ciment présente très peu de couleurs avec une domination de l'ocre rouge composée d'un mélange d'oxyde de fer $\left(\mathrm{Fe}_{2} \mathrm{O}_{3}\right)$ et d'argiles. Avec l'ocre jaune (qui est également un oxyde de fer) et un pigment noir (oxyde de manganèse), ces trois pigments sont les seuls détectés par les analyses en diffraction des rayons $\mathrm{X}$ et en microspectrométrie Raman. Grâce à la coloration naturelle du ciment gris, les chevaux, imposants sur la fresque de saint Hippolyte, ne sont travaillés qu'au niveau des ombres et des contours noirs.

La fresque sur chaux du chemin de croix de Fourqueux en revanche possède une palette colorée plus fournie. En plus des oxydes de fer et de manganèse déjà cités, s'ajoute un bleu dérivé du précieux Lapis-Lazuli et synthétisé au $\mathrm{XX}^{\mathrm{e}}$ siècle, le bleu outremer. On retrouve également un vert de chrome (oxyde de chrome, $\mathrm{Cr}_{2} \mathrm{O}_{3}$ ) ainsi qu'un blanc de titane (dioxyde de titane, $\mathrm{TiO}_{2}$ ), caractéristique des pigments modernes. La réalisation et l'étude de stratigraphies au sein du laboratoire montrent le pouvoir colorant de certains pigments. Ainsi, difficilement détectables, les grains d'oxydes de fer se répartissent au niveau de la couche picturale de façon hétérogène et discontinue mais laissent pourtant une impression visuelle de continuité de couleur.

L'observation en microscopie électronique à balayage des coupes stratigraphiques montre bien l'insertion du pigment dans les premiers micromètres sous l'effet mécanique du pinceau et de la rhéologie de l'enduit. Pourtant, les échantillons prélevés sur le chemin de croix de Fourqueux montrent une délimitation nette entre l'enduit support et une couche picturale également fournie en calcite, élément de l'enduit (fig. $\mathbf{n}^{\circ} \mathbf{1 1}$ ). Cette observation, compte tenu de la technique de préparation de surface - lissage et usage de l'éponge favorise l'hypothèse d'un liant à l'eau de chaux. Cette eau riche en ions active ainsi la tenue de la fresque tout en apportant des teintes plus pastel. 
Figure 11

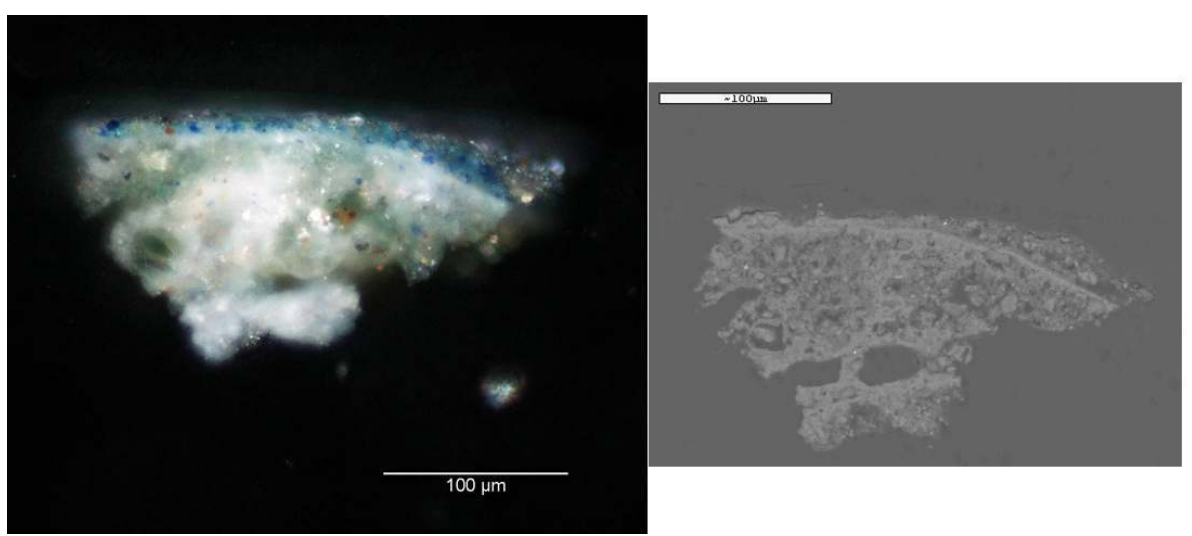

Stratigraphie d'un échantillon du Chemin de Croix de Fourqueux sous microscopies optique et électronique à balayage (mode électrons rétrodiffusés), délimitation nette entre les couches picturales et l'enduit support. 11a: Coupe observée en microscopie optique. 11b: Coupe observée en microscopie électronique à balayage, mode électrons rétrodiffusés.

(c) Bayle, Marine/C2RMF, LCR, 2011.

La comparaison des couches picturales des fresques sur ciment de saint Hippolyte et sur chaux avec le Chemin de Croix montre des similitudes en termes d'insertion des pigments; ceux-ci sont noyés dans les premiers micromètres. Très fines, jusqu'à $2 \mu \mathrm{m}$ d'épaisseur sur enduit de ciment, les couches picturales de Marret sont assez irrégulières. Si la fresque sur chaux présente des superpositions de couleurs, la fresque sur ciment ne possède qu'une seule couche colorée. Traditionnellement, la superposition de couleurs à fresque permet de donner de la profondeur et des contrastes plus facilement. La technique des drapés telle que l'enseigne Cennini dans son Traité des Arts ${ }^{21}$, s'effectue en apposant en premier la couleur opposée à celle souhaitée. Pour un drapé bleu outremer, Marret peint ses ombres en rouge. Un exemple de panneau montre la vierge Marie, traditionnellement représentée vêtue de bleu dans la religion, portant un drapé rose. L'étude microscopique des prélèvements indique la présence de bleu outremer en surface. Avec le temps, le pigment bleu a très certainement dû se détériorer laissant ainsi apparaitre la couche sous-jacente (fig. $\mathbf{n}^{\circ} \mathbf{1 2}$ ). Des tracés bleus sont encore visibles aux endroits forts du drapé permettant ainsi de conserver la structure et le mouvement du vêtement. 
Figure 12

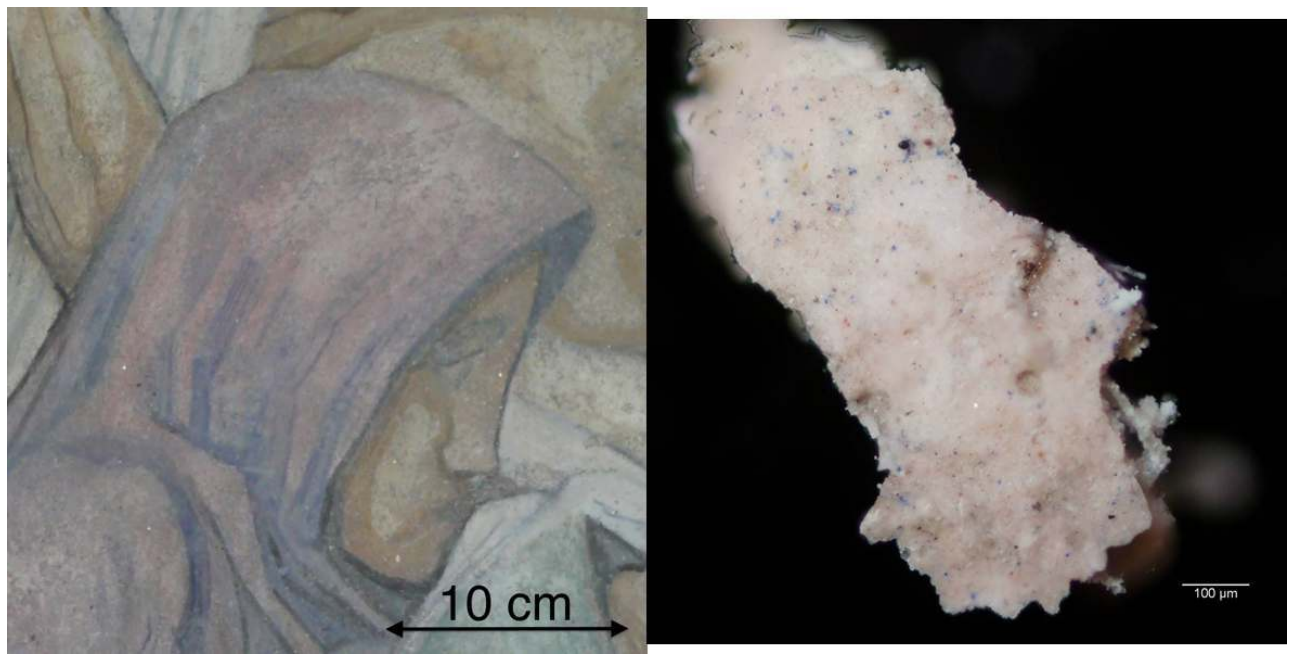

Détail du panneau XIII où Marie est représentée vêtue de rose et observation plus précise d'un échantillon prélevé sur la partie haute du vêtement (tête). 12a : Détail de la tunique de Marie. 12b : Surface de l'échantillon prélevé sur la tunique observée en microscopie optique.

Phot. Bayle, Marine. (c) Bayle, Marine/C2RMF, LCR, 2011.

La fresque sur enduit de ciment présente elle-aussi une particularité de technique. Les stratigraphies étudiées présentent les caractéristiques microstructurales particulières du gypse, en aiguilles. La micro-diffraction des rayons $\mathrm{X}$ a pu établir avec certitude la présence de ce composé en surface des échantillons (fig. $\mathbf{n}^{\circ}{ }^{13}$ ). 
Figure 13

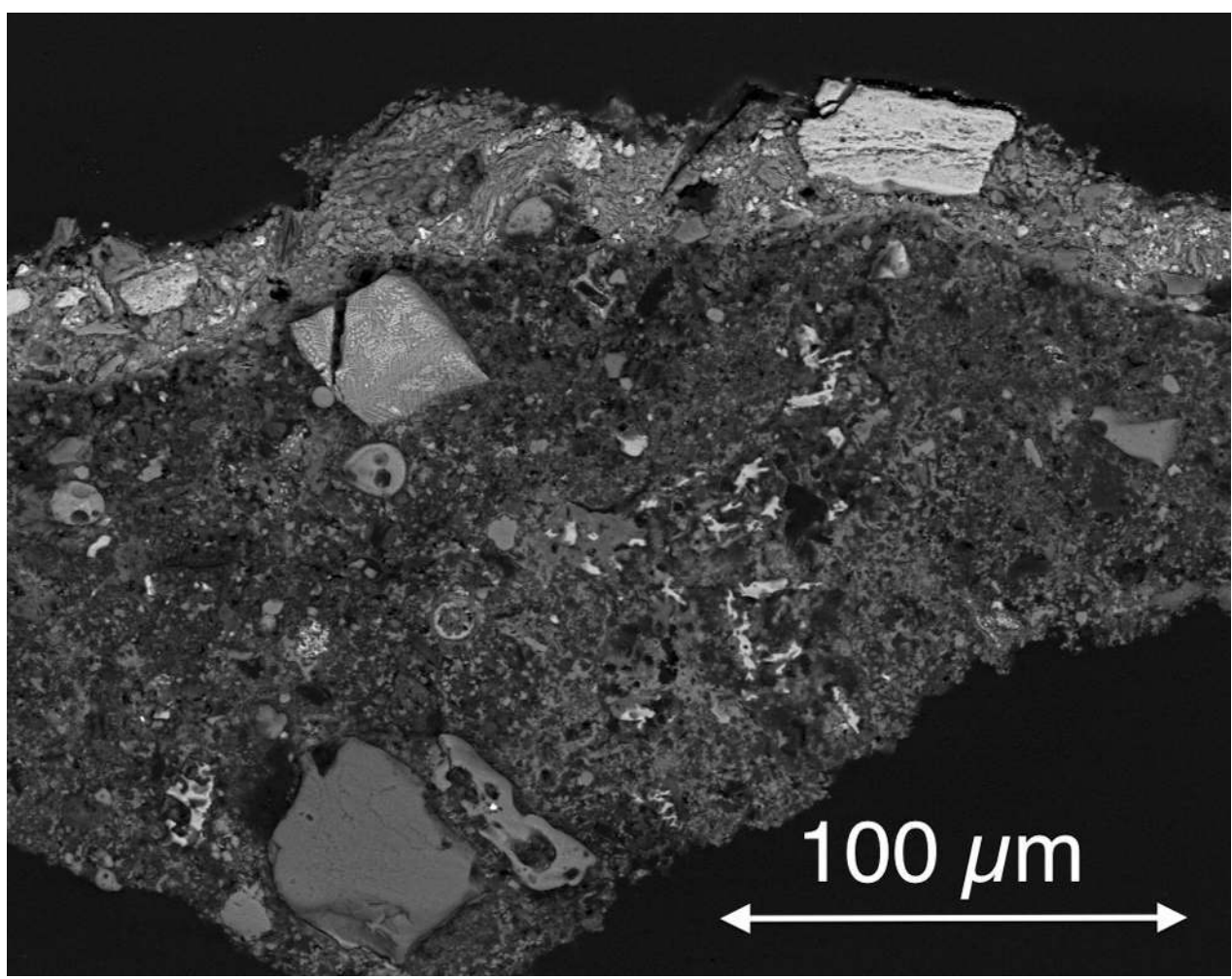

Microstructure en aiguilles de la couche picturale de la fresque des Martyrs de saint Hippolyte et de saint Laurent, observée en microscopie électronique à balayage, mode électrons rétrodiffusés.

(c) Bayle, Marine/C2RMF, LCR, 2011.

Henri Marret travaillait rapidement, les tracés sont secs et les formes des personnages sont géométriques. La présence de couches picturales superposées sur le Chemin de Croix de l'église de Fourqueux peut laisser penser que le temps de travail a été favorisé par les délais de prise de la chaux, laissant le loisir de travailler la couleur avant durcissement complet de la surface. Comme évoqué précédemment, la fresque sur ciment ne présente qu'une seule couche colorée mais chargée en gypse. Étant donné les concentrations de gypse détectées, il est certain que cette application était volontaire mais le but du fresquiste reste indéterminé : Marret a-t-il utilisé le gypse telle une eau de chaux pensant favoriser la pérennité de la fresque ? Ou, connaissant peut-être de manière empirique les propriétés de ce composé ajouté en faible quantité sur l'hydratation du ciment, il a choisi de l'utiliser pour ralentir la prise de surface?

\section{Comparaison des œuvres de Marret avec les reconstitutions effectuées en atelier}

L'étude des fresques de Marret a été complétée par une série de reconstitutions en atelier grâce à la collaboration de Mme Delahousse, artiste fresquiste <lien vers www.solenedelahousse.fr>. Ces essais ont permis de renforcer les hypothèses formulées au cours de l'analyse des œuvres à la fois pour la réalisation artistique mais aussi pour la technique de la fresque sur ciment (fig. $\left.\mathbf{n}^{\circ} \mathbf{1 4}\right)$. 
Figure 14

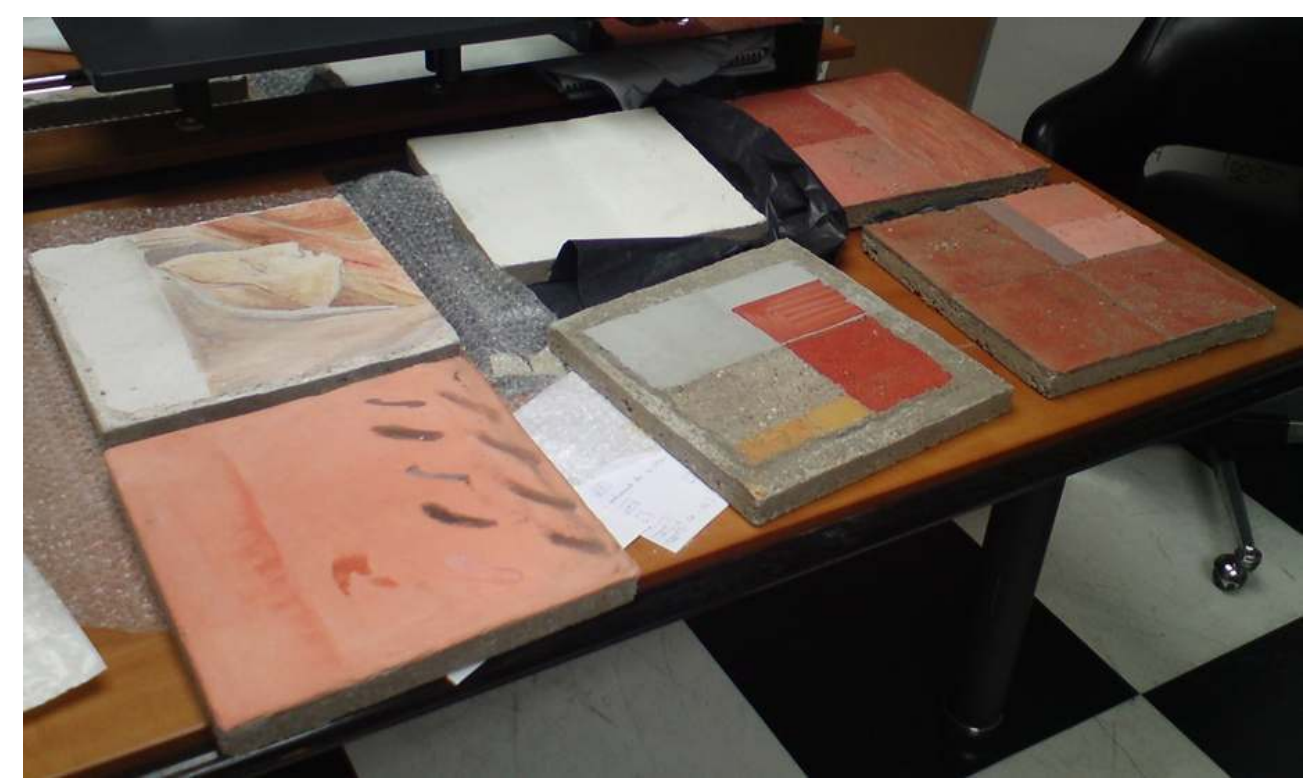

Ensemble des panneaux réalisés à fresque sur chaux ou sur ciment lors de la phase de reconstitution. Phot. Gueit, Éléonore, 2011. (c) É. Gueit.

L'accroche des pigments ainsi que leur tenue sur l'enduit de ciment est possible en appliquant la technique ancestrale de fresque. L'utilisation de gypse n'est donc pas théoriquement utile, l'insertion mécanique des grains de pigments dans les premiers micromètres de l'enduit, sous l'effet du pinceau, est suffisante pour obtenir une œuvre à fresque. Le ciment est plus difficile à travailler avant d'appliquer les pigments, mais le temps de prise reste tout à fait raisonnable pour un travail précis et réfléchi de la couleur. La fresque sur chaux sera plus longue à durcir mais, pour un artiste comme Marret, travaillant vite et expérimenté, les temps d'application des pigments sont relativement similaires sur chaux comme sur ciment (fig. $\left.\mathbf{n}^{\circ} \mathbf{1 5}\right)$. 
Figure 15

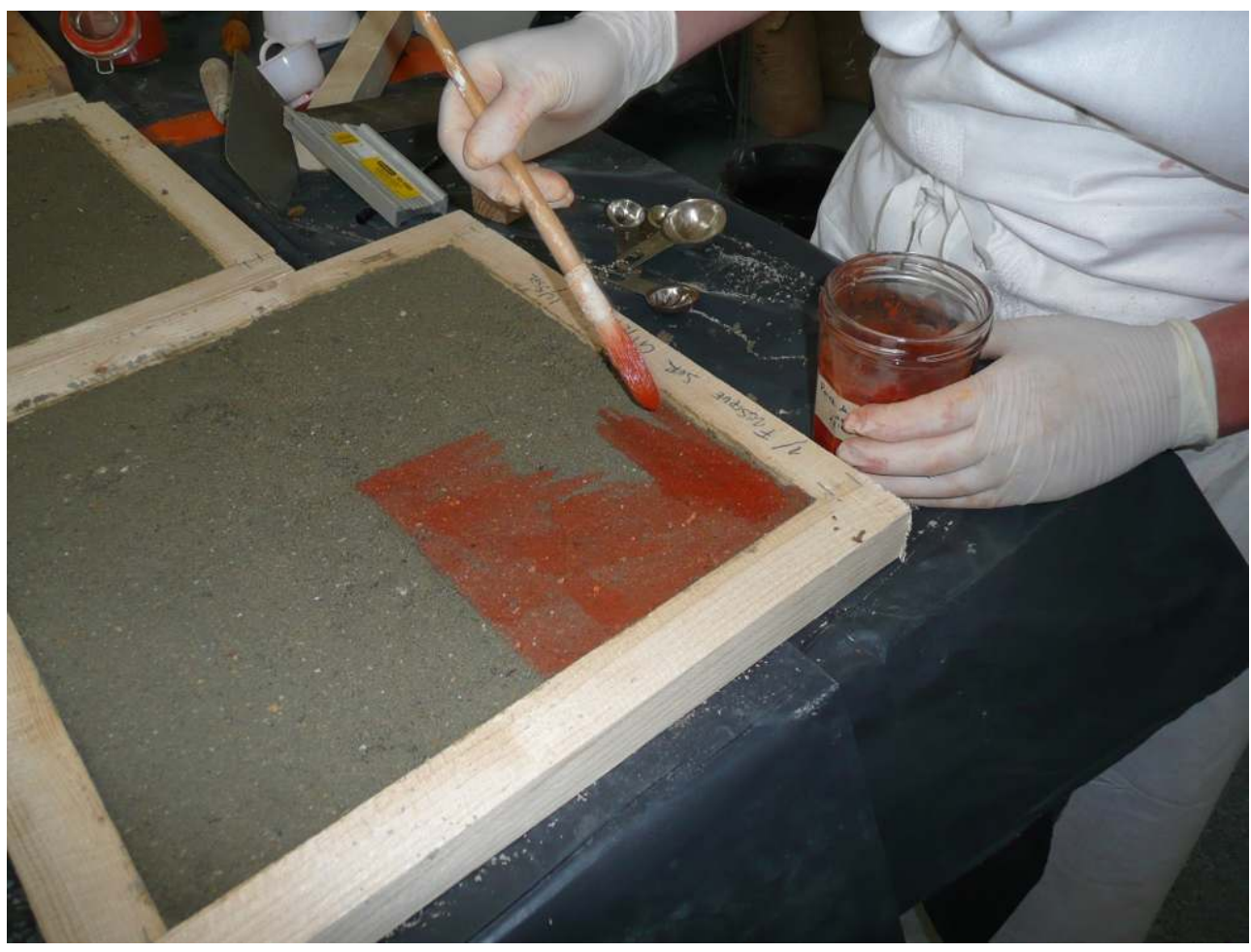

Application au pinceau de pigments rouges (ocre rouge) en suspension aqueuse sur un enduit de ciment frais. Solène Delahousse.

Phot. Bayle, Marine, 2011. (c) M. Bayle.

Le panneau reconstitué à fresque sur ciment, selon la technique des pigments liés à l'eau, montre qu'aucune migration de gypse en surface ne s'effectue alors que les essais réalisés à l'eau de plâtre permettent d'obtenir une stratigraphie totalement en accord avec l'œuvre étudiée. D'autre part, en étant beaucoup plus épaisses $(100 \mu \mathrm{m})$, les couches picturales observées montrent que la finesse de la fresque de saint Hippolyte n'est pas particulièrement liée à l'hydratation du ciment mais que Marret a ainsi choisi d'appliquer une couche picturale fine laissant apparaître la profondeur grise du ciment support.

Les comparaisons des essais de lissage sur ciment et sur chaux corroborent bien la difficulté soulevée par Georges Lecaron ${ }^{22}$. En effet, lorsque le ciment est travaillé en surface à la taloche comme pourrait le faire un artiste sur chaux, l'outil provoque des décollements de l'intonaco et l'effet de la prise rapide de l'enduit gêne le travail du fresquiste ; peu accentué, il adoucit le grain de surface et rappelle les détails observés sur la fresque de saint Hippolyte (auréoles, visages). Cette action n'est pas aux fondements des mécanismes de fixation des pigments mais elle procure des possibilités artistiques intéressantes, tout particulièrement sur chaux.

Enfin, les conclusions proposées sur la technique de fresque sur chaux de Marret (Chemin de Croix de Fourqueux) lors de l'étude préalable ont été appuyées par la reproduction effectuée par Mme Delahousse. Le lissage de l'arricio et le nettoyage de la surface à l'éponge avant application des pigments permettent d'obtenir une fresque qui présente les caractéristiques artistiques observées à la surface de l'original. 


\section{Influence de la carbonatation sur l'adhésion des pigments?}

34 carbonatation de la chaux sous l'effet du dioxyde de carbone atmosphérique. Bien évidemment, cette réaction joue un rôle important dans la pérennité de la fresque en apportant une dureté de surface et une stabilité chimique qui ont permis de garder certaines des fresques du début de notre ère dans un état exceptionnel de conservation. Si les romains maîtrisaient cette réaction de carbonatation par des effets de lissages des enduits et/ou de la couche picturale, permettant la cristallisation de calcite par une remontée d'eau en surface, cette étape n'apporte à la fresque qu'un renforcement et un aspect esthétique différent. L'ancrage des pigments s'effectue par un travail mécanique qui induit une certaine pénétration dans l'extrême surface de la pâte fraîche. La technique sur ciment montre tout particulièrement que la carbonatation agit beaucoup trop lentement et tardivement pour avoir un impact sur l'adhésion des pigments.

\section{Conclusion}

Les mécanismes de fixation à fresque des pigments sont identiques sur chaux comme sur ciment. L'étude comparative de deux œuvres d'un même artiste, Henri Marret, a permis de montrer que les grains de pigments s'insèrent mécaniquement dans l'enduit frais grâce à l'eau provenant des couches sous-jacentes et du pinceau lors de l'application des couches picturales. L'utilisation d'un liant organique gênerait cette insertion tout en menaçant la pérennité de la fresque. Henri Marret a effectué des choix artistiques particuliers pour fixer les pigments en respectant les caractéristiques des matériaux employés ainsi que la technique ancestrale de peinture à fresque. Ces artistes, qui ont su maîtriser les avancées technologiques et industrielles du début du XXe siècle nous ont offert des œuvres magnifiques laissant ainsi perdurer la technique de fresque. Ce renouveau de l'art de la fresque ouvre ainsi des possibilités de décoration picturale dans les travaux modernes. Pourtant plus cher et plus difficile à travailler que la chaux, le ciment, grâce à des artistes passionnés, a permis de faire briller une des plus anciennes techniques de peinture murale au temps des avancées industrielles.

\section{NOTES}

1. - DELAHOUSSE, Solène, ROBIC, Yves. La fresque un décor à la chaux. Massin, 2009. <lien vers www.solenedelahousse.fr>.

2. - DELAHOUSSE, Solène, ROBIC, Yves. Op. cit.

3. - DELAHOUSSE, Solène, ROBIC, Yves. Op. cit.

4. - VITRUVE. De Architectura, livre VII, 2, 4, traduit par C. Perrault, 1684. Pierre Mardaga, 1995.

5. - PLINE L'Ancien. Naturalis Historiae, XXXV-XXXVI, traduit par H. Zehnacker. Paris : Les BellesLettres, CUF, 1983. 
6. - CENNINI, Cennino. Il libro del Arte, Traité des Arts, traduit par Victor Mottez, traduction de 1911. L'œil d'Or, 2009.

7. - DELAHOUSSE, Solène, ROBIC, Yves. Op. cit. CENNINI, Cennino, Op. cit.

8. - GUEIT, Éléonore. Coloration superficielle du béton frais via l'agent de décoffrage, comparaison avec la technique de peinture à fresque. Thèse. Centre de Mise en forme des Matériaux : Mines-Paris Tech 2012. <lien vers http://pastel.archives-ouvertes.fr/pastel-00686903>.

9. - GUEIT, Éléonore. Thèse. Centre de Mise en forme des Matériaux : Mines-Paris Tech 2012. Op. cit.

10. - SIQUEIROS, David Alfaro. L'art et la révolution : Réflexions à partir du muralisme mexicain. Paris : Éditions sociales, 1973.

11. - MAGNE, Henri. « La peinture à fresque sur ciment ». Bulletin de la Société d'Encouragement pour l'Industrie Nationale, 1923, p. 79-84.

12. - GUEIT, Éléonore. Op. cit.

13. - PIQUE, F., DERRICK, M., PARKER, A., SCOTT, D.A., SCHILLING, D. and SAAVEDRA PEREZ, M. "Original technique of the mural America Tropical by David Alfaro Siqueiros". Materials Research Society Symposium Proceedings, 1995, 352, p. 365-371.

14. - MAGNE, Henri. Op. cit.

15. - PIQUE, F., DERRICK, M., PARKER, A., SCOTT, D.A., SCHILLING, D. and SAAVEDRA PEREZ, M. Op. cit.

16. - MARRET, Henri. «Peinture à fresque sur chaux ou ciment ». L'âge du Ciment, bulletin technique de la Société Anonyme des Chaux et Ciments de Lafarge et du Teil, 1937, 10, p. 33-36.

17. - HALL, Christopher. "On the History of Portland Cement after 150 years". Journal of Chemical Education, 1976, 53/4, p. 222-223.

18. - TAYLOR, H.F.W. Cement Chemistry, (2 ed.). London: Thomas Telford Publishing, 1997.

19. - LECARON, Georges. "Essai sur des techniques de peinture: la fresque ». L'information Culturelle Artistique, Nov-Dec 1956, 5.

20. - TAYLOR, H.F.W. Op. cit.

21. - CENNINI, Cennino. Op. cit.

22. - LECARON, Georges. Op. cit.

\section{RÉSUMÉS}

Au cours de l'histoire, la technique de peinture à fresque, largement employée dans le monde occidental, tout particulièrement sous l'époque Romaine, est connue pour être la plus durable des peintures murales. La technique traditionnelle consiste à appliquer les pigments en suspension dans l'eau sur un enduit frais à la chaux qui, peu après, durcit par réaction de carbonatation. Suite à l'invention du ciment Portland au $\mathrm{XX}^{\mathrm{e}}$ siècle, certains artistes muralistes ont expérimenté une technique similaire sur enduit de ciment. Bien que les mécanismes de prise du ciment soient différents de ceux de la chaux, les fresques sur ciment présentent un aspect et une durabilité identiques aux fresques sur chaux. Les aspects historiques et techniques de la fresque sur chaux sont bien documentés, mais les mécanismes fondamentaux responsables des propriétés de surface ont rarement été étudiés et restent encore peu compris. Fondée sur l'analyse de fresques du début $\mathrm{du} \mathrm{XX}^{\mathrm{e}}$ siècle ainsi que sur des reconstitutions en laboratoire, cette étude apporte de nouveaux éléments sur la formation des surfaces de fresques. 
The fresco painting technique has been widely used throughout history in the occidental world, in particular at the Roman period, and is known to be the most durable form of mural painting. The traditional technique consists in applying water-dispersed pigments on a fresh lime plaster, which later hardens through carbonation reaction. Following the invention of the Portland cement at the $20^{\text {th }}$ century, some mural artists experimented a similar painting technique on cement plaster. Although the setting mechanisms of cement are different from lime, the cement frescoes present the same aspect and the same durability as the lime frescoes. The historical and practical aspects of the lime technique are well-documented, but the fundamental mechanisms responsible for the properties of a fresco surface have rarely been investigated and are still poorly understood. Based on analyses of frescoes from the early $20^{\text {th }}$ century and on laboratory reconstitutions, this study brings new insight on the formation of a fresco surface.

\section{INDEX}

Keywords : fresco, Henri Marret, lime, cement, surface, pigments

Mots-clés : fresque, Henri Marret, chaux, ciment, surface, pigments, « Martyrs de saint Hippolyte et de saint Laurent ", Chemin de croix de Fourqueux

\section{AUTEURS}

\section{MARINE BAYLE}

Ingénieur en Sciences des Matériaux, C2RMF - Centre de Recherche et de Restauration des Musées de France. Doctorante en Physique et Chimie des Matériaux, LAPA - Laboratoire Archéomatériaux et Prévision de l'Altération, CEA/CNRS UMR 3299 marine.bayle@cea.fr

\section{ÉLÉONORE GUEIT}

Docteur en Sciences des Matériaux à l'École des Mines de Paris, CEMEF - Centre de Mise en Forme des Matériaux, CNRS UMR 7635 eleonore.gueit@hotmail.fr

\section{ÉVELYNE DARQUE-CERETTI}

Docteur d'État ès Sciences, groupe «Surfaces et Tribologie », CEMEF - Centre de Mise en Forme des Matériaux evelyne.darque-ceretti@mines-paristech.fr

\section{MATTHIEU HORGNIES}

Docteur en science des Matériaux à l'École des Mines de Paris, chargé de recherche au Laboratoire central de recherche de Lafarge (LCR) matthieu.horgnies@lafarge.com

\section{MARC AUCOUTURIER}

Ingénieur civil des Mines, directeur de recherche émérite au CNRS, chercheur au Centre de Recherche et de Restauration des Musées de France (C2RMF) marc.aucouturier@culture.gouv.fr 